\title{
Personalized Context Aware Assignment Recommendations in E-Learning System
}

\author{
M. Venu Gopalachari \\ Assistant Professor \\ Dept of CSE \\ CBIT, Hyderabad
}

\author{
P. Sammulal, $\mathrm{PhD}$ \\ Assistant Professor \\ Dept of CSE \\ JNTUH college of Engg., Jagitial
}

\begin{abstract}
E-Learning is the technology that targets the community seeks learning through various means such as material, information, courses to facilitate the ease of access. Online delivery of educational instruction provides the opportunity to bring colleges and universities new energy, students, and revenues. However e-learning system has rapid development in making activities of learning online and providing a vast set of resources for the material and online assignments to complete. Although personalized e-learning systems developed and provides services they limited to focus their recommendations of the material is only about the student's level interest of surfing on the learning material but never considered the level of understanding of the learning material. This system developed a model that aim to recommend the assignments and material of the course to the student based on the level of understanding by analyzing the performance of the student in past. Experimental results on the proposed recommender system exhibited significant results than the traditional elearning system. This shown the impact of personalized assignment recommendations in improving the student's interest towards the course.
\end{abstract}

\section{Keywords}

Classification, e-learning, Recommender System, Web Mining.

\section{INTRODUCTION}

The advancements in distributed and parallel computing in networks and exponential growth in technology of web services lead the learning procedure effective and flexible especially education became a new brink in this era. Typically e-learning system is the collection of various technologies and methods to provide knowledge of education or training electronically with the combination of traditional ad advanced managing techniques [15]. Several different approaches have been developed to deliver online education in an academic setting. One of the key areas where data mining can be used to fetch lucrative results is E- Learning. Web-based educational technologies allow educators to evaluate student's performance and which learning strategies are most effective $[14,16]$. The importance of learner profile is getting focused in many aspects of improving the efficiency of activities in elearning. Most of these consider the unformatted information such as course names, course materials to be the sole needy thing in learning. But all of the existing strategies lack of personalization towards a particular user in terms of evaluation and training. For instance an assignment given to a set of students used to be common which observes all of them as same level of ability towards the course. If everyone given a sophisticated assignment then it is obvious for set of students with less ability to attempt or else if the assignment is simple enough for all then there is no way for the set of students who have high ability towards course to get a chance to prove. In both of these cases the assignments does not help the entire set of students to improve their knowledge. Since web-based educational systems are able to find the patterns of learner profile, data mining and knowledge discovery techniques can be applied to find interesting relationships between attributes of students, their performance and apply these relationships to recommend assignments personalized to the individual students. In this paper a model is developed that aims to solve the following:

- Recommend assignments to individual students by means of personalization based on their past performance with an aim to improve their knowledge according to their ability to take it.

- Provide the faculty with a way to visualize a student's performance and a way to design the efficient assignments.

The fuzziness in the marks and deadline of the assignments is defined with predefined labels by the classification technique. The experiments made with the proposed e-learning recommender system shown a significant impact on the student's growth in terms of ability to taking the course rather than the traditional web based e-learning system.

\section{RELATED WORK}

Now a day various electronic resources such as e-commerce, e-governance, digital libraries, social network sites leads to the information overload problem. Recommendation Systems for a specific domain are the trend choice to reduce this problem to its maximum extent by suggesting probably relative pages to the user $[11,12]$. One of the fields that got affected by the information overload problem is education or e-learning domain. For instance the student in e-learning system surely gets confused to get material from enormous size of the documents available. In that situation definitely the recommenders helps to sort out the preferences of the user and makes the user comfortable by suggesting relatively relevant material.

Electronically supported learning that aims to construct proper knowledge with respect to all is called as e-learning. It generally uses the networked media or computer stored knowledge base for learning. E-learning considers all of its information as the input for the process of the mining and report generation for the activities provided.

The e-learning initiative was launched by the European Commission to encourage its spread. There is also an increased use of virtual classrooms as an online learning platform and classroom for a diverse set of education 
providers. Apart from the advancements in network technologies such as virtual classroom environments, social networks have become one of the important means in Elearning $[10,13]$. Also social networks are acting as platform to share information in the process of learning by means of subjective discussions and educational purposes. E-learning systems can cover multiple types of applications as in[2] to improve the quality of the system as well the satisfaction of the learner.

E-learning with recommender systems is the upcoming trend in learning process with formatted and unformatted information. Recommenders can be either content based [7] that focus on the user's profile based on usage history, collaborative filtering [9] that focus on behavior of similar users or hybrid recommender [4] that mix up the advantages of both content based and collaborative filtering. Maatallah and et.al pinned the problem of stability of technology to recommending by enhancing the $\mathrm{CF}$ methodology using learner's interests [5]. J. Bobadilla et. al proposed CF technique for e-leaning based on the knowledge level of the user as a parameter [11]. Some methods focused on the ratings of the learners to identify the sequential patterns of the user in addition with attributes of material to improve the quality of learning[3]. This framework extracts latent patterns of the user's access for materials and for which constructs the tree with the learner's interests. In [6] a methodology using association analysis that gives association patterns of user profile is proposed that suggests the activities based on the user's behavior. J. Lu proposed a framework that recommends material information of a subject to the user with personalization by making use of the history and also mentioned to deal with the fuzziness in the ratings given by the user [2]. But these recommenders didn't consider the evaluation strategy in recommending information.

\section{ASSIGNMENT RECOMMENDER SYSTEM}

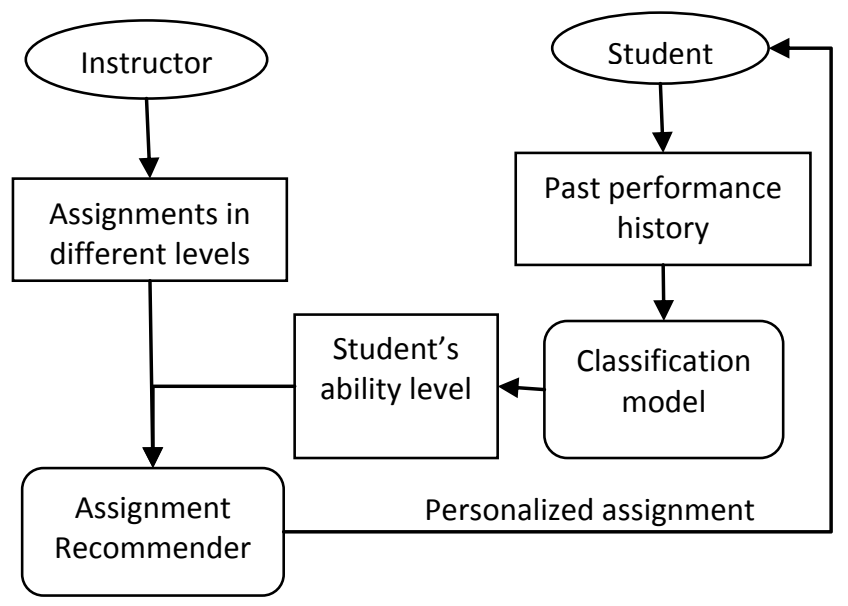

Figure 1: Architecture of proposed e-learning recommender system.

This research prime motivation is to keep track of the student's performance and also the level of design of the test or assignment has to be synchronized with the level of learning ability about that course. . In a normal web based education system, users are involved with two kinds of large data sets: 1) educational resources such as web pages, demonstrations, simulations, and individualized problems designed for use on homework assignments, quizzes, and examinations; and 2) information about users who create, modify, assess, or use these resources. In other words, we have two ever-growing pools of data. As the resource pool grows, the information from students who have multiple transactions with these resources also increases. The system logs any access to these resources as well as the sequence and frequency of access in relation to the successful completion of any assignment.

The proposed model is developed for students within such systems, to help the students to utilize the resources and help instructors design the assignments efficiently as shown in figure1. The key step of this model is applying classification model on the student's performance history so that to define the learning ability with a predefined labels such as poor, average or excellent etc. However the assignments are uploaded with different levels of difficulty such as easy, moderate and difficult instead of keeping common level of assignment. There after a mapping model that maps assignments at different levels to the students with different levels based on the predefined conditions by the instructor.

This model tries to prove the feasibility of using Recommendation Systems applications in educational environments. A Recommendation System is an application capable of presenting a user a suggestion for an object, obtained on the basis of user's previous preferences and the preferences of a community which has likings and opinions similar to the user interests.

The recommendation system would recommend a learning task to a learner based on the tasks already done by the learner and their successes. In principle, there are two major parts in the design of a learning agent: a "learning" module that learns from past access patterns and infers an individual or common access model; and an "advising" module that applies the learned model at given times to recommend actions.

The system is implemented as different phases: instructor phase, student phase, recommendation phase and visualization phase.

\section{Instructor phase:}

- Faculty will enter the login details and gets access to the website.

- Faculty will upload the particular subject assignment and will mention the assignment deadlines.

- If any student submits the assignment, the faculty should evaluate it and award marks.

Student Phase:

- Once the student gains access to the website he can check the assignments of the particular subjects followed by its deadlines.

- The student will submit answers to the assignment.

- Once the marks are awarded, the student can view his marks.

Recommendation phase:

- The assignments uploaded by the instructor usually contain three sets with ' $n$ ' number of questions in each of them. The sets include easy, medium and tough questions. 
- The first assignment is assigned randomly to all the students.

- Once the student submits his assignment, they are evaluated and marks are given. Combining both marks and the number of days before which the student has submitted the answer, grades are given. Grades are assigned using "Decision Tree Induction" Algorithm.

The next assignment to the student is based on his grade in previous assignment. For example, if a student scores grade "Poor" in an assignment, the next time he will be given an easy task, giving him a chance to improve his fundamentals.

\section{Visualization phase:}

- Faculty can view a student's performance, subject wise.

- Once a faculty enters a class, section and subject a list of student of that section are displayed. On clicking the link, details of the student from the database is copied to visualizing tool, converting it to a graph displays the student's performance assignment wise.

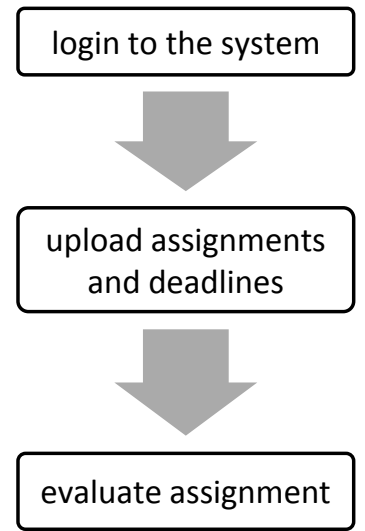

(a)

apply classification on all users

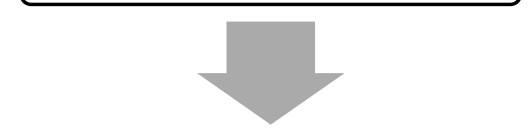

Assign grades to all users

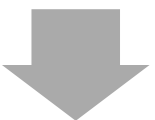

Map various levels of assignments to defferent users

(c)

Figure 2: The proposed system with different user's perspective a) instructor view b) student view c) Recommender view.
As explained above, this model is basically to improve the performance and knowledge of a student with the help of assignments. Firstly a faculty logs into the portal and uploads an assignment for a subject by entering certain details like class, section, assignment number, deadline etc. Then he uploads the file, which gets stored in the database. Later, whenever any student logs in, he can see all the assignments which were assigned to him along with the deadlines. The student can then click on any assignment, to view the question and also submit the answer.

Faculty has a chance to check if any student has submitted the answers and evaluate them. Using the rules generated from the ID3 algorithm mentioned below, for the combination of marks and lvalue (number of days before or after the deadline the student has submitted the assignment) grades are assigned to the students. Here some predefined class labels for the student used are excellent, average and poor. For example if a student is graded excellent, then for the next assignment, he gets a tough question or else if the student graded as poor then system recommends easy question. For instance, the following model snippet explains the rules depicting how the grade of student allotted based on his previous performance as well the lvalue derived from the deadline of the assignment.

$$
\begin{aligned}
& \text { if (lval }>=0 \text { and } \text { mar }>=8) \\
& \text { grade }={ }^{\prime} E^{\prime} \\
& \text { if (lval }>0 \text { and } l v a l<=4 \text { and } \text { mar }>=5 \text { and } \text { mar }<8 \text { ) } \\
& \text { grade }={ }^{\prime} A^{\prime} \\
& \text { if (lval }>0 \text { and } l v a l<=6 \text { and } \text { mar }>=0 \text { and } \operatorname{mar}<5) \\
& \text { grade }={ }^{\prime} P^{\prime} \\
& \text { if (lval }>4 \text { and } l v a l<=6 \text { and } \text { mar }>=5 \text { and } \operatorname{mar}<8) \\
& \text { grade }={ }^{\prime} E^{\prime} \\
& \text { if }(\text { lval }>=-6 \text { and lval }<=-2) \\
& \text { grade }={ }^{\prime} P^{\prime} \\
& \text { if (lval }=-1 \text { and } \text { mar }>=8) \\
& \text { grade }=^{\prime} A \text { ' } \\
& \text { if (lval }=-1 \text { and mar }>5 \text { and } \operatorname{mar}<8) \\
& \text { grade }=^{\prime} P^{\prime}
\end{aligned}
$$

Now the mapping of assignments and students are made as such if the student is with ' $E$ ' grade then will get a difficult question, and if the student is with ' $\mathrm{P}$ ' grade then will get an easy question. However difficult question will get more weightage in awarding marks than easy question even they have same lvalue means that for example, if student ' $A$ ' got difficult question and answered within deadline having lvalue $=2$ and student ' $B$ ' got easy question and answered within deadline having same lvalue $=2$ then the student ' $A$ ' will get more marks than student ' $\mathrm{B}$ '. The weightage difference is pre defined by the instructor for all levels of the assignment.

\section{RESULTS AND ANALYSIS}

An e-learning portal is implemented with proposed model observed the final performance of the students on set of courses. The motto of the experimentation is finding the overall improvement of the student and number of students in each ability label. Three class labels for the students have considered they are Excellent (E), Average (A) and Poor (P). The experiments were carried with a set of courses and with a 
set of assignments for each course. After experimentation the percentage of students in each class label is recorded and compared with the data without recommendation model.

The performance of e-learners after providing recommendations is significantly improved as shown in figure 3. Most of the students with poor performance are moved to average performance. As recommendations are more useful to slow learners, the overall learning effectiveness is improved. The accuracy of learning recommendations is defined as percentage of learners followed the recommended page. Thus, assignment recommendation accuracy represents the usefulness of assignment recommendations while learning.

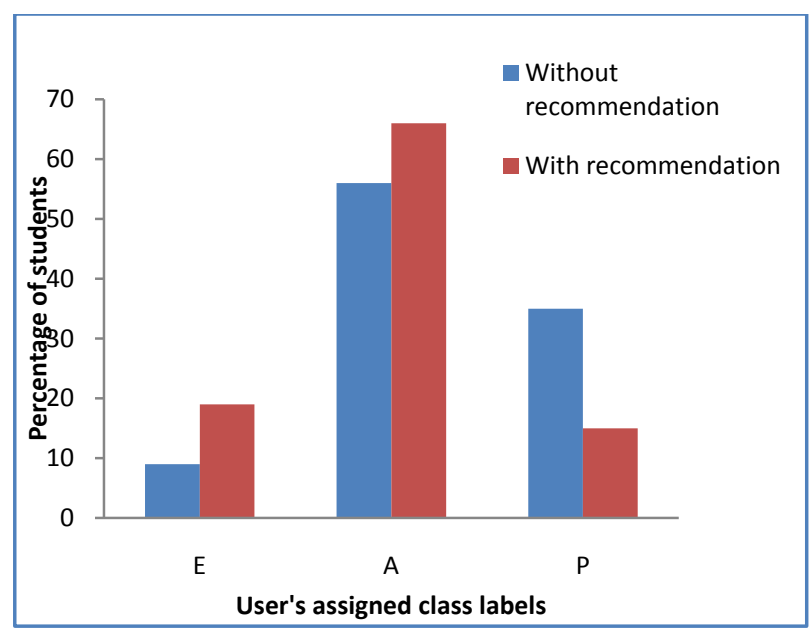

Figure3: comparison of percentage of students with various class labels with and without proposed recommendation strategy.

The proposed model is also compared with an existing recommender proposed in [11] so as to describe the effectiveness of the proposed model. In order to this Movie lens dataset is considered even though it is not an e-learning dataset because it is used in existing method. And to analyze the performance the measure MAE (Mean Absolute Error) is used where it processes the expected and actual ratings of suggestions.

Table 1: MAE values for different set of training data

\begin{tabular}{|l|l|l|}
\hline & $\begin{array}{l}60 \% \text { training } \\
\text { data }\end{array}$ & $\begin{array}{l}80 \% \text { training } \\
\text { data }\end{array}$ \\
\hline $\begin{array}{l}\text { Existing } \\
\text { Recommender }\end{array}$ & 0.842 & 0.811 \\
\hline $\begin{array}{l}\text { Proposed } \\
\text { Model }\end{array}$ & 0.757 & 0.723 \\
\hline
\end{tabular}

The experiments made by changing the training set percentage with test data set and the MAE values are in table1. This says the proposed model has shown efficiency rather than existing model.

\section{CONCLUSION}

As the Web based education systems have pools of data resources, which are growing at a faster pace, making use of this rapidly growing data, there is a wide scope to improve the concept of E-Learning. This paper addresses the issues surrounding the use of a data mining framework within a web based educational system. This system developed a recommender model that considers the previous performance in order to assign a task that balances the ability of the user and the hardness of the task. It provides a chance to the users in e learning system to improve their performance gradually and also there is an ample scope for the instructor to design the assignments effectively with a view to help students gain more knowledge about the subject. It also provides the faculty an option to view a student's performance graphically.

\section{REFERENCES}

[1] Dianshuang Wu, Jie Lu, Guangquan Zhang, "A Fuzzy Tree Matching-based Personalized eLearning Recommender System", IEEE Transactions on Fuzzy Systems, 2015.

[2] J. Lu, "Personalized e-learning material recommender system", in Proceedings of the 2nd International Conference on Information Technology for Application (ICITA 2004), 2004, pp. 374-379.

[3] M. Salehi and I. N. Kamalabadi, "Hybrid recommendation approach for learning material based on sequential pattern of the accessed material and the learner's preference tree", Knowledge Based Systems, volume 48, pp. 57-69, 2013

[4] M. Salehi and I. N. Kmalabadi, "A hybrid attributebased recommender system for e-learning material recommendation", IERI Procedia, volme 2, pp. 565-570, 2012.

[5] M. Maâtallah and H. Seridi, "Enhanced collaborative filtering to recommender systems of technology enhanced learning," in ICWIT 2012, 2012, pp. 129-138.

[6] O. R. Zaiane, "Building a recommender agent for elearning systems", in Proceedings of 2002 International Conference on Computers in Education, 2002, pp. 55-59

[7] Hafidh Ba-Omar, I. P. “A Framework for Using Web Usage Mining to Personalise E-learning. Seventh IEEE International Conference on Advanced Learning Technologies (ICALT 2007) (pp. 937-938). Japan, 2007.

[8] Hai-Jew, "Scaffolding Discovery Learning Spaces. MERLOT Journal of Online Learning and Teaching, $p$. 533-549, USA, 2008

[9] Cheqian Chen, K. L. "Personalized search based on learning user click history", Cognitive Informatics (ICCI), 2010 9th IEEE International Conference on (pp. 490-495), Beijing, 2010

[10] Kosuke Takano,"An Adaptive e- Learning Recommender Based on User's Web- Browsing Behavior", International Conference on P2P, Parallel, Grid, Cloud and Internet Computing (pp. 978-0-76954237-9/10). IEEE, 2010.

[11] J. Bobadilla, F. Serradilla, and A. Hernando, "Collaborative filtering adapted to recommender systems of e-learning", Knowledge-Based Systems, volume 22, pp. 261-265, 2009

[12] Marta Zorrilla, S. M., "Data Web house to support web intelligence in e-learning environments. Granular Computing", IEEE International Conference (pp. 722 727 Vol. 2). Spain, 2005.

[13] Mohamed Koutheair Khribi, M. J, "Automatic Recommendations for E-Learning Personalization Based on Web Usage Mining Techniques and Information Retrieval”, Eighth IEEE International Conference on 
Advanced Learning Technologies pp. 30-42, Washington, DC, USA, 2008.

[14] Olfa Nasraoui, M. S, “ A Web Usage Mining Framework for Mining Evolving User Profiles in Dynamic Web Sites", IEEE Transactions on Knowledge and data engineering, VOL. 20, NO. 2, February 2008 (pp. 10414347/08). IEEE, 2008
[15] Xinjin Li, S. Z, "Application of Web Usage Mining in elearning Platform", International Conference on EBusiness and E-Government, IEEE, 2010.

[16] Xue Sun, W. Z, “ Design and Implementation of an Elearning Model Based on WUM Techniques", International Csonference on E-Learning, EBusiness, Enterprise Information Systems, and EGovernment, IEEE, 2009. 